\title{
Optimal Color Filter Array Design: Quantitative Conditions and an Efficient Search Procedure
}

\author{
Yue M. Lu and Martin Vetterli \\ Audio-Visual Communications Laboratory \\ School of Computer and Communication Sciences \\ Swiss Federal Institute of Technology Lausanne (EPFL), Lausanne, Switzerland
}

\begin{abstract}
Most digital cameras employ a spatial subsampling process, implemented as a color filter array (CFA), to capture color images. The choice of CFA patterns has a great impact on the performance of subsequent reconstruction (demosaicking) algorithms. In this work, we propose a quantitative theory for optimal CFA design. We view the CFA sampling process as an encoding (low-dimensional approximation) operation and, correspondingly, demosaicking as the best decoding (reconstruction) operation. Finding the optimal CFA is thus equivalent to finding the optimal approximation scheme for the original signals with minimum information loss. We present several quantitative conditions for optimal CFA design, and propose an efficient computational procedure to search for the best CFAs that satisfy these conditions. Numerical experiments show that the optimal CFA patterns designed from the proposed procedure can effectively retain the information of the original full-color images. In particular, with the designed CFA patterns, high quality demosaicking can be achieved by using simple and efficient linear filtering operations in the polyphase domain. The visual qualities of the reconstructed images are competitive to those obtained by the state-of-the-art adaptive demosaicking algorithms based on the Bayer pattern.
\end{abstract}

\section{INTRODUCTION}

As a cost-effective way to capture color images, most digital cameras employ a single monochromatic image sensor, in combination with a color filter array (CFA). Placed directly in front of the sensor array, the CFA performs a spatial subsampling procedure, in which every pixel in the array records one portion of the incoming light spectrum. A key process in the digital imaging pipeline is thus to reconstruct full-resolution color images from their CFA downsampled versions. This interpolation problem, often called demosaicking (or demosaicing), has received considerable attention, with numerous algorithms proposed in the literature (see Refs. 1-3 for excellent reviews on existing work).

The choice of CFA patterns used in the acquisition process has a great impact on the performance of various demosaicking algorithms. Although the Bayer patten, ${ }^{4}$ invented in the mid-1970s, remains the most widely used CFA arrangement, a flurry of new CFA designs (e.g. Refs. 5-7) have been proposed recently, aiming at providing improved reconstruction quality with simplified demosaicking processes. In particular, the recent work of Hirakawa and Wolfe ${ }^{7}$ has demonstrated that, by carefully designing the CFA pattern, it is possible to achieve high quality demosaicking with simple linear filtering operations.

In this work, we propose a quantitative theory for optimal CFA design. Our discussions build upon several previous work ${ }^{8-10}$ on demosaicking, which approaches the problem as a linear minimum mean square error (LMMSE) reconstruction process. We further the above line of thinking by studying the following question: given the LMMSE demosaicking scheme, what is the optimal CFA pattern that minimizes the reconstruction error? To answer the above question, it is helpful to reinterpret the CFA sampling operation from a different perspective. Instead of being a "passive" downsampling (or degradation) process, we can think of the CFA sampling as an "active" encoding (low-dimensional approximation) operation and, correspondingly, demosaicking as the

Further author information: (Send correspondence to Y. M. Lu.)

Y. M. Lu: yue.lu@epfl.ch; M. Vetterli: martin.vetterli@epfl.ch

Digital Photography V, edited by Brian G. Rodricks, Sabine E. Süsstrunk,

Proc. of SPIE-IS\&T Electronic Imaging, SPIE Vol. 7250, $725009 \cdot$ • 2009 SPIE-IS\&T

CCC code: 0277-786X/09/\$18 - doi: 10.1117/12.807598

SPIE-IS\&T/ Vol. 7250 725009-1 


\begin{tabular}{|c|c|c|}
\hline $\begin{array}{ll}G & R \\
B^{1} & G\end{array}$ & $\begin{array}{ll}G & R \\
B^{4} & G\end{array}$ & $\begin{array}{ll}\mathrm{G} & \mathrm{R} \\
\mathrm{B}^{7} & \mathrm{G}\end{array}$ \\
\hline $\begin{array}{ll}G & R \\
B & \\
& \\
& \\
G\end{array}$ & $\begin{array}{ll}G & R \\
B^{5} & G\end{array}$ & $\begin{array}{l}G \quad R \\
B^{8} G\end{array}$ \\
\hline $\begin{array}{ll}G & R \\
B^{3} & G\end{array}$ & $\begin{array}{ll}G & R \\
B^{6} & G\end{array}$ & $\begin{array}{l}G \quad R \\
B^{9} G\end{array}$ \\
\hline
\end{tabular}

Figure 1. A portion of the Bayer CFA, whose sampling pattern is 2-by-2 periodic. Each of the nine periodic blocks contains two green pixels, one red pixel, and one blue pixel.

best decoding (reconstruction) operation. Finding the optimal CFA is thus equivalent to finding the optimal approximation scheme for the original signals with minimum information loss.

There exist close analogies between the above task and several classical problems in communication and learning theories, for which the Karhunen-Loève transform, also referred to as the principle component analysis (PCA), ${ }^{11}$ provides the optimal solutions. The challenge in our case comes from the physical setups of the CFA. In particular, we need to search for the optimal CFA among a very limited subset of possible "encoding" matrices. This additional constraint greatly complicates the optimization problem and precludes simple solutions provided by the PCA.

The rest of the paper is organized as follows. We first briefly overview the concept of optimal (minimum mean square error) linear demosaicking in Section 2, which serves as the starting point of our discussion. Interpreting the CFA sampling processes as constrained low-dimensional approximation operators, we provide in Section 3 several equivalent quantitative conditions for optimal CFA design. The associated optimization task turns out to be nontrivial. In Section 4 we propose an efficient iterative procedure to search for the optimal CFAs. Numerical experiments in Section 5 show that the optimal CFA patterns designed from the proposed procedure can effectively retain the information of the original full-color images. In particular, with the designed CFA patterns, high quality demosaicking can be achieved by using simple and efficient linear filtering operations in the polyphase domain. Section 6 concludes the paper. We present in this paper only the main results and ideas of our work, and leave all the proofs and other technical details to Ref. 12.

\section{PRELIMINARIES}

\subsection{The Bayer CFA and its Matrix-Vector Representation}

We start by describing a general matrix-vector notation for the CFA sampling process. Although for simplicity reasons our discussion in this section focuses solely on the Bayer CFA, ${ }^{4}$ the formulations presented below can be easily generalized (see Section 3.2) to work with arbitrary periodic CFAs.

As shown in Figure 1, the sampling pattern of the Bayer CFA is 2 -by-2 periodic. For the $k$ th $(1 \leq k \leq 9)$ block in the figure, we can define a corresponding "super pixel" ${ }^{8}$ to be the following vector

$$
\boldsymbol{x}_{k} \stackrel{\text { def }}{=}\left[r_{k, 1}, r_{k, 2}, r_{k, 3}, r_{k, 4}, g_{k, 1}, g_{k, 2}, g_{k, 3}, g_{k, 4}, b_{k, 1}, b_{k, 2}, b_{k, 3}, b_{k, 4}\right]^{T},
$$

where $\left\{r_{k, n}, g_{k, n}, b_{k, n}\right\}_{n=1}^{4}$ are the red, green, and blue pixels in that block. Due to the CFA sampling process, the sensor can only observe a partial version of $\boldsymbol{x}_{k}$. Denoting by $\boldsymbol{y}_{k}$ the vector of sensor measurements on the $k$ th block, we have

$$
\boldsymbol{y}_{k} \stackrel{\text { def }}{=}\left[g_{k, 1}, b_{k, 2}, r_{k, 3}, g_{k, 4}\right]^{T} \text {. }
$$

The observation $\boldsymbol{y}_{k}$ and the full-resolution super pixel $\boldsymbol{x}_{k}$ can be linked in a compact form as $\boldsymbol{y}_{k}=\boldsymbol{A}_{0} \boldsymbol{x}_{k}$, where

$$
\boldsymbol{A}_{0} \stackrel{\text { def }}{=}\left[\begin{array}{lll}
\boldsymbol{P}_{r} & \boldsymbol{P}_{g} & \boldsymbol{P}_{b}
\end{array}\right]
$$


is a block matrix containing three diagonal submatrices

$$
\boldsymbol{P}_{r} \stackrel{\text { def }}{=} \operatorname{diag}(0,0,1,0), \boldsymbol{P}_{g} \stackrel{\text { def }}{=} \operatorname{diag}(1,0,0,1), \text { and } \boldsymbol{P}_{b} \stackrel{\text { def }}{=} \operatorname{diag}(0,1,0,0) .
$$

\subsection{Linear Minimum Mean Square Error Demosaicking: A Brief Overview}

We only consider linear demosaicking algorithms in this paper. Despite their simplicity and nonadaptive nature, linear algorithms enjoy great advantages in computational efficiency. Moreover, their filtering-based implementations are especially suitable for hardware realizations.

Suppose that we want to estimate the full-resolution super pixel $\boldsymbol{x}_{5}$ in Figure 1 through a linear combination of all sensor measurements $\left\{\boldsymbol{y}_{1}, \ldots, \boldsymbol{y}_{9}\right\}$ within its 3-by-3 neighborhood. This estimation process can be represented by the following matrix-vector product

$$
\widehat{\boldsymbol{x}}_{5}=\boldsymbol{D}\left[\boldsymbol{y}_{1}^{T}, \boldsymbol{y}_{2}^{T}, \ldots, \boldsymbol{y}_{9}^{T}\right]^{T},
$$

where $\widehat{\boldsymbol{x}}_{5}$ is the estimated value and $\boldsymbol{D}$ is the demosaicking matrix.

Adopting the notational convention introduced in Ref. 10, we stack all the super pixels within the 3-by-3 neighborhood and get

$$
\boldsymbol{p}_{5} \stackrel{\text { def }}{=}\left[\boldsymbol{x}_{1}^{T}, \boldsymbol{x}_{2}^{T}, \ldots, \boldsymbol{x}_{9}^{T}\right]^{T} .
$$

In what follows, we refer to such stacked vectors as "hyperpixels". Since $\boldsymbol{y}_{k}=\boldsymbol{A}_{0} \boldsymbol{x}_{k}$ for all $k$, it follows that

$$
\left[\boldsymbol{y}_{1}^{T}, \boldsymbol{y}_{2}^{T}, \ldots, \boldsymbol{y}_{9}^{T}\right]^{T}=\boldsymbol{A} \boldsymbol{p}_{5}
$$

where

$$
\boldsymbol{A} \stackrel{\text { def }}{=} \boldsymbol{I}_{9} \otimes \boldsymbol{A}_{0} .
$$

We call the matrix $\boldsymbol{A}$ defined above the CFA sampling matrix.

The quality of reconstruction can be measured in terms of the squared distance between the original signal $\boldsymbol{x}_{5}$ and its estimate $\widehat{\boldsymbol{x}}_{5}\left(=\boldsymbol{D} \boldsymbol{A} \boldsymbol{p}_{5}\right)$ as follows

$$
\begin{aligned}
e_{5} & =\left\|\boldsymbol{x}_{5}-\widehat{\boldsymbol{x}}_{5}\right\|^{2} \\
& =\left\|\boldsymbol{S} \boldsymbol{p}_{5}-\boldsymbol{D} \boldsymbol{A} \boldsymbol{p}_{5}\right\|^{2},
\end{aligned}
$$

where $\boldsymbol{S} \stackrel{\text { def }}{=}[0,0,0,0,1,0,0,0,0] \otimes \boldsymbol{I}_{12}$ effectively "selects" $\boldsymbol{x}_{5}$ from $\boldsymbol{p}_{5}$. If we have access to a dictionary of $K$ hyperpixels $\left\{\boldsymbol{p}_{k}\right\}_{k=1}^{K}$, then the average performance of a demosaicking matrix $\boldsymbol{D}$ can be measured by the mean square error of reconstruction, defined as

$$
M S E \stackrel{\text { def }}{=} \frac{1}{K} \sum_{k=1}^{K}\left\|\boldsymbol{S} \boldsymbol{p}_{k}-\boldsymbol{D} \boldsymbol{A} \boldsymbol{p}_{k}\right\|^{2} .
$$

The optimal linear demosaicking scheme in the minimum mean square error (MMSE) sense is thus the solution to the following optimization problem: ${ }^{8-10}$

$$
\boldsymbol{D}^{*}=\underset{\boldsymbol{D}}{\arg \min } \frac{1}{K} \sum_{k=1}^{K}\left\|\boldsymbol{S} \boldsymbol{p}_{k}-\boldsymbol{D} \boldsymbol{A} \boldsymbol{p}_{k}\right\|^{2} .
$$

A closed form solution to (8) is given by the classical Wiener estimator

$$
\boldsymbol{D}^{*}=\boldsymbol{S} \boldsymbol{C} \boldsymbol{A}^{T}\left(\boldsymbol{A C A ^ { T } ) ^ { \dagger } ,}\right.
$$

where

$$
\boldsymbol{C} \stackrel{\text { def }}{=} \frac{1}{K} \sum_{k=1}^{K} \boldsymbol{p}_{k} \boldsymbol{p}_{k}^{T}
$$

is the data correlation matrix. 


\section{QUANTITATIVE CONDITIONS FOR OPTIMAL CFA DESIGN}

\subsection{CFA Sampling as Optimal Low-Dimensional Approximations}

In this work, we study the following question: given the LMMSE demosaicking scheme in (8), what is the optimal CFA pattern (i.e. sampling matrix $\boldsymbol{A}$ ) that minimizes the reconstruction error? More specifically, we aim to solve the following constrained optimization problem

$$
\boldsymbol{A}^{*}=\underset{\boldsymbol{A} \in \mathcal{A}}{\arg \min }\left(\min _{\boldsymbol{D}} \frac{1}{K} \sum_{k=1}^{K}\left\|\boldsymbol{S} \boldsymbol{p}_{k}-\boldsymbol{D} \boldsymbol{A} \boldsymbol{p}_{k}\right\|^{2}\right),
$$

where $\mathcal{A}$ is a set containing all feasible CFAs patterns. There exist close analogies between the above task and several classical problems in communication and learning theories. In particular, it is helpful to interpret the CFA sampling matrix $\boldsymbol{A}$ in (11) as an encoding (low-dimensional approximation) operation and, correspondingly, demosaicking $\boldsymbol{D}$ as the best decoding (reconstruction) operation. Finding the optimal CFA is thus equivalent to finding the optimal approximation scheme for the original signals with minimum information loss.

The challenge in our case arises from the physical setups of the CFA, which confine our search within a very limited set $\mathcal{A}$ of possible "encoding" matrices. This additional constraint greatly complicates the optimization problem and precludes classical solutions such as the Karhunen-Loève transform (or PCA).

\subsection{Matrix-Vector Representations for General CFAs}

In order to precisely define the constraint set $\mathcal{A}$ in (11), we first need to extend the notation introduced in Section 2.1 to the general cases of periodic CFA patterns of block size $N$-by- $N$, where $N \geq 2$.

Similar to (1), we can define a super pixel $\boldsymbol{x}_{k}$ (of size $3 N^{2} \times 1$ ) for each $N$-by- $N$ block. Meanwhile, within each neighborhood of $(2 L+1)$-by- $(2 L+1)$ super pixels, we can define the stacked hyperpixel $\boldsymbol{p}_{k}$ as in $(5)$. The CFA sampling process can again be represented as a matrix-vector multiplication. The corresponding CFA sampling matrix is obtained as

$$
\boldsymbol{A}=\boldsymbol{I}_{(2 L+1)^{2}} \otimes \boldsymbol{A}_{0},
$$

where $\boldsymbol{A}_{0}$ is defined in (2). In the general case, the submatrices $\boldsymbol{P}_{r}, \boldsymbol{P}_{g}, \boldsymbol{P}_{b}$ of $\boldsymbol{A}_{0}$ are diagonal matrices of size $N$-by- $N$, written as

$$
\boldsymbol{P}_{r}=\operatorname{diag}\left(c_{r}(1), \ldots, c_{r}\left(N^{2}\right)\right), \boldsymbol{P}_{g}=\operatorname{diag}\left(c_{g}(1), \ldots, c_{g}\left(N^{2}\right)\right), \text { and } \boldsymbol{P}_{b}=\operatorname{diag}\left(c_{b}(1), \ldots, c_{b}\left(N^{2}\right)\right),
$$

respectively. The "color coefficient vector"

$$
\boldsymbol{c} \stackrel{\text { def }}{=}\left[c_{r}(1), \ldots, c_{r}\left(N^{2}\right), c_{g}(1), \ldots, c_{g}\left(N^{2}\right), c_{b}(1), \ldots, c_{b}\left(N^{2}\right)\right]^{T}
$$

represent the relative percentages of red, green, and blue information acquired at different pixels in the CFA block. Unlike the Bayer CFA for which the color coefficients can only take values of either 0 or 1 , we allow the color coefficients to be real numbers, subject to the following convexity condition

$$
\boldsymbol{c} \in \mathcal{C} \stackrel{\text { def }}{=}\left\{\boldsymbol{c}: c_{r}(n) \in[0,1], c_{g}(n) \in[0,1], c_{b}(n) \in[0,1], c_{r}(n)+c_{g}(n)+c_{b}(n)=1, \text { for } 1 \leq n \leq N^{2}\right\} .
$$

The CFA sampling matrix $\boldsymbol{A}$ is completely determined by the color coefficient vector $\boldsymbol{c}$. In fact, from its definition, we can represent $\boldsymbol{A}$ as a function of $\boldsymbol{c}$ as

$$
\boldsymbol{A}(\boldsymbol{c})=\sum_{n=1}^{N^{2}}\left(c_{r}(n) \boldsymbol{M}_{r}(n)+c_{g}(n) \boldsymbol{M}_{g}(n)+c_{b}(n) \boldsymbol{M}_{b}(n)\right),
$$

where $\left\{\boldsymbol{M}_{r}(n), \boldsymbol{M}_{g}(n), \boldsymbol{M}_{b}(n)\right\}_{n}$ are constant matrices of zeros and ones. The constraint set $\mathcal{A}$ can now be compactly defined as

$$
\mathcal{A} \stackrel{\text { def }}{=}\{\boldsymbol{A}(\boldsymbol{c}): \boldsymbol{c} \in \mathcal{C}\}
$$




\subsection{Three Equivalent Conditions for Optimal CFA Design}

Note that the data correlation matrix $\boldsymbol{C}$ defined in (10) is positive semidefinite. Consequently, we can factorize it as $\boldsymbol{C}=\boldsymbol{P P}$, where $\boldsymbol{P}$ is another positive semidefinite matrix. The "square root" matrix $\boldsymbol{P}$ turns out to be very useful in simplifying the optimization condition given in (11).

LEMma 3.1. The mean square error term in (7) can be rewritten as

$$
M S E=\left\|\boldsymbol{P} \boldsymbol{S}^{T}-\boldsymbol{P} \boldsymbol{A}^{T} \boldsymbol{D}^{T}\right\|_{F}^{2},
$$

where $\|\cdot\|_{F}$ is the Frobenius norm of a matrix.

Substituting (13) into (11), we reach our first condition on optimal CFAs.

Proposition 1. The optimal CFA can be obtained as

$$
\boldsymbol{A}^{*}=\underset{\boldsymbol{A} \in \mathcal{A}}{\arg \min }\left(\min _{\boldsymbol{D}}\left\|\boldsymbol{P} \boldsymbol{S}^{T}-\boldsymbol{P} \boldsymbol{A}^{T} \boldsymbol{D}\right\|_{F}^{2}\right) .
$$

The above mean square error is minimized when $\boldsymbol{D}$ is equal to

$$
\boldsymbol{D}^{*}(\boldsymbol{A})=\left(\boldsymbol{P} \boldsymbol{A}^{T}\right)^{\dagger} \boldsymbol{P} \boldsymbol{S}^{T}
$$

Replacing $\boldsymbol{D}$ in (14) with $\boldsymbol{D}^{*}(\boldsymbol{A})$, we can obtain the second equivalent condition for optimal CFA design as follows.

Proposition 2. The optimal CFAs can be obtained by

$$
\boldsymbol{A}^{*}=\underset{\boldsymbol{A} \in \mathcal{A}}{\arg \min }\left\|\left(\boldsymbol{I}-\left(\boldsymbol{P} \boldsymbol{A}^{T}\right)\left(\boldsymbol{P} \boldsymbol{A}^{T}\right)^{\dagger}\right) \boldsymbol{P} \boldsymbol{S}^{T}\right\|_{F}^{2} .
$$

We note that $\boldsymbol{I}-\left(\boldsymbol{P} \boldsymbol{A}^{T}\right)\left(\boldsymbol{P} \boldsymbol{A}^{T}\right)^{\dagger}$ in (16) is in fact the projection onto the subspace that is orthogonal to $\mathcal{R}\left(\boldsymbol{P} \boldsymbol{A}^{T}\right)$. Denoting this projection operator by $\mathbf{P}_{\mathcal{R}\left(\boldsymbol{P} \boldsymbol{A}^{T}\right)^{\perp} \text {, we can further simplify the equivalent condition for }}$ optimal CFA as follows.

Proposition 3. The optimal CFAs can be obtained by

$$
\boldsymbol{A}^{*}=\underset{\boldsymbol{A} \in \mathcal{A}}{\arg \min } \sum_{n=1}^{3 N^{2}}\left\|\mathbf{P}_{\mathcal{R}\left(\boldsymbol{P} \boldsymbol{A}^{T}\right)^{\perp}} \boldsymbol{p}_{n}\right\|^{2},
$$

where $\boldsymbol{p}_{n}\left(1 \leq n \leq 3 N^{2}\right)$ is the nth column vector of $\boldsymbol{P} \boldsymbol{S}^{T}$.

The above result leads to a nice geometric interpretation for optimal CFA design. Intuitively, by changing the CFA sampling matrix $\boldsymbol{A}$, we can "tilt" the subspace $\mathcal{R}\left(\boldsymbol{P} \boldsymbol{A}^{T}\right)$. Finding the optimal CFA is thus equivalent to finding the best "tilted" subspace that are closest to the point cloud $\left\{\boldsymbol{p}_{n}\right\}_{n=1}^{3 N^{2}}$ in orthogonal distances.

\section{AN EFFICIENT COMPUTATIONAL PROCEDURE FOR OPTIMAL CFA DESIGN}

As shown in Section 3.2, the CFA sampling matrices $\boldsymbol{A} \in \mathcal{A}$ can be parameterized by the color coefficient vectors $\boldsymbol{c} \in \mathcal{C}$. Consequently, (17) is equivalent to minimizing a penalty function

$$
f(\boldsymbol{c}) \stackrel{\text { def }}{=} \sum_{n=1}^{3 N^{2}}\left\|\mathbf{P}_{\mathcal{R}\left(\boldsymbol{P} \boldsymbol{A}^{T}(\boldsymbol{c})\right)^{\perp}} \boldsymbol{p}_{n}\right\|^{2}
$$

of $3 N^{2}$ variables. Unfortunately, such functions are generally nonconvex. For example, we show in Figure 2 a 2 -D cross section of such a function, which exhibits highly nonconvex behaviors. 


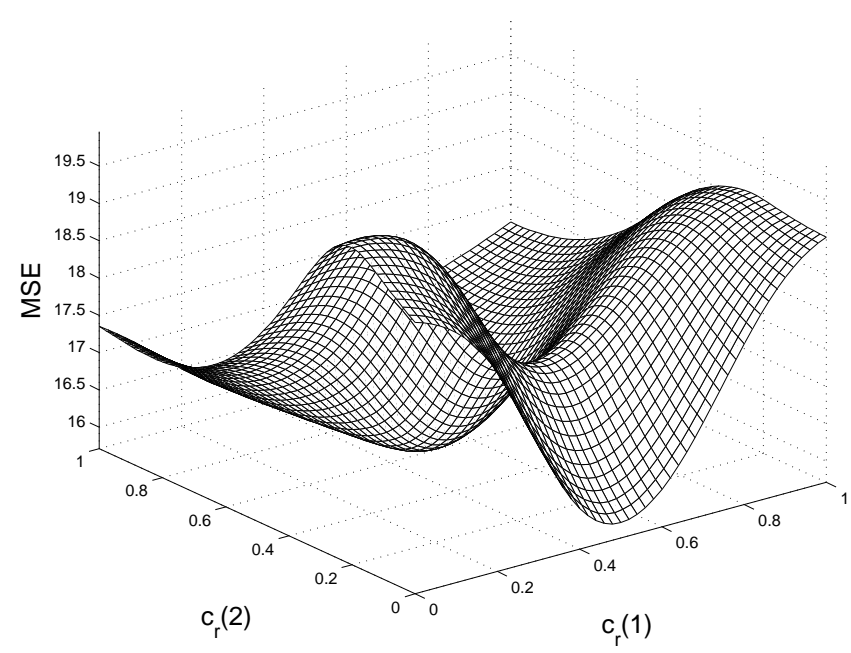

Figure 2. A 2-D cross section of the penalty function $f(\boldsymbol{c})$.

In what follows, we propose an iterative procedure to search for the optimal CFAs. Each step in the proposed iteration process is a convex optimization problem with easy solutions. To start, we first rewrite the "sequential" optimization problem in (14) into the following "simultaneous" optimization scheme

$$
\left(\boldsymbol{A}^{*}, \boldsymbol{D}^{*}\right)=\underset{\boldsymbol{A} \in \mathcal{A}, \boldsymbol{D}}{\arg \min }\left\|\boldsymbol{P} \boldsymbol{S}^{T}-\boldsymbol{P} \boldsymbol{A}^{T} \boldsymbol{D}\right\|_{\mathrm{F}}^{2} .
$$

For fixed $\boldsymbol{A}$, the above problem is convex, and the corresponding optimal solution $\boldsymbol{D}^{*}(\boldsymbol{A})$ is given in (15). Now consider the case when we fix $\boldsymbol{D}$ and search for the best $\boldsymbol{A}$. Substituting (12) into (18), we get

$$
\boldsymbol{c}^{*}(\boldsymbol{D})=\underset{\boldsymbol{c} \in \mathcal{C}}{\arg \min }\left\|\boldsymbol{P} \boldsymbol{S}^{T}-\sum_{n=1}^{N^{2}}\left(c_{r}(n) \boldsymbol{P} \boldsymbol{M}_{r}^{T}(n) \boldsymbol{D}+c_{g}(n) \boldsymbol{P} \boldsymbol{M}_{g}^{T}(n) \boldsymbol{D}+c_{b}(n) \boldsymbol{P} \boldsymbol{M}_{b}^{T}(n) \boldsymbol{D}\right)\right\|_{\mathrm{F}}^{2} .
$$

This is a quadratic programming problem with inequality constraints, which can be efficiently solved by methods such as the interior point algorithm. ${ }^{13}$ We can now summarize the proposed iterative search procedure as follows.

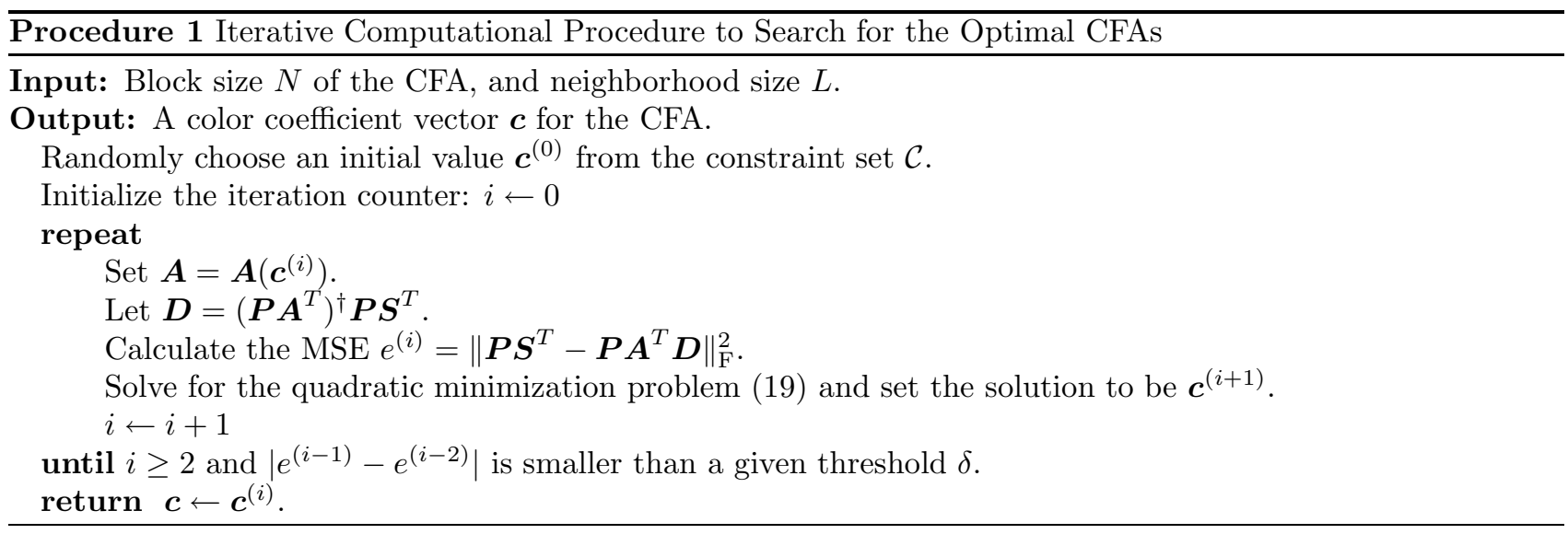

\section{NUMERICAL EXPERIMENTS}

In our experiments, we set the parameters to be $N=4$ and $L=2$. This choice means that we are searching for 4-by-4 CFA patterns, and that the demosaicking algorithm can be implemented as 5-by-5 linear filtering 


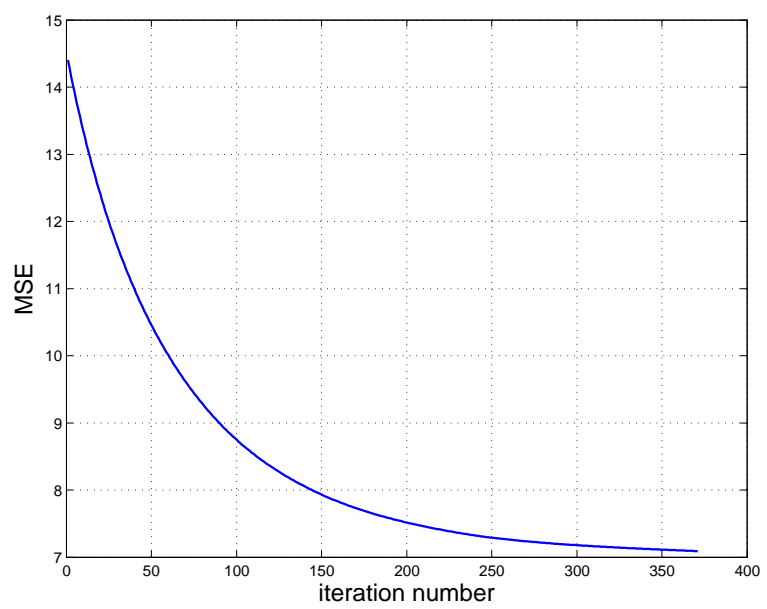

(a)

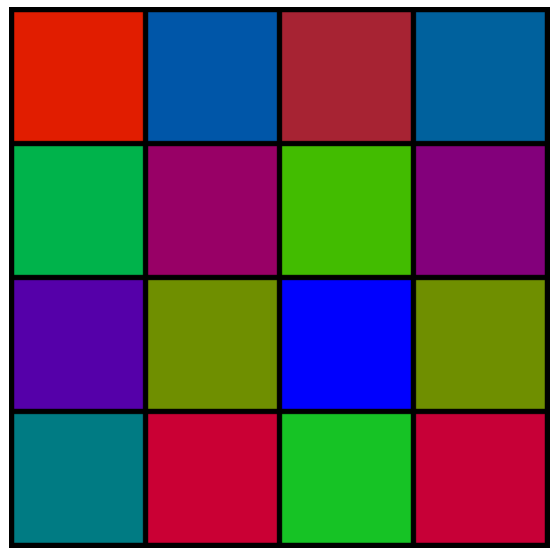

(b)

Figure 3. (a) The convergence behavior of the proposed iterative search procedure. (b) The obtained 4-by-4 periodic CFA.

operations in the polyphase domain. We calculate the data correlation matrix $\boldsymbol{C}$ in (10) by using a database of 24 standard Kodak test images. Figure 3(a) shows the convergence behavior of the proposed iterative search procedure. We can see that the MSE values decrease monotonically throughout the iteration process, and the entire procedure converges after about 360 iterations. The result of the search is a 4 -by-4 CFA, whose sampling pattern is shown in Figure 3(b).

Figure 4 shows the detailed portions of six color images reconstructed from the 4-by-4 CFAs designed by the proposed scheme. Note that in obtaining these results, we adopt the standard "leave-one-out" method: we obtain the CFA by calculating the data correlation matrix $\boldsymbol{C}$ from 23 out of the 24 test images; we then test the CFA and the demosaicking algorithm on the image that was left out. The demosaicking process uses simple nonadaptive linear filtering in the polyphase domain. Despite the relative simplicity of the demosaicking algorithm, the reconstructed images show fairly high qualities with few visible artifact, which suggests the effectiveness of the proposed optimal CFA design procedure.

\section{CONCLUSIONS}

We proposed a quantitative theory for optimal CFA design. The key in our work is to interpret the CFA sampling operation as an "active" encoding (low-dimensional approximation) operation and, correspondingly, demosaicking as the LMMSE decoding (reconstruction) operation. Finding the optimal CFA is thus equivalent to finding the optimal approximation scheme for the original signals with minimum information loss. We presented several equivalent conditions for optimal CFA design, and proposed an efficient iterative procedure to search for the best CFAs that satisfy these conditions. Numerical experiments show that the optimal CFA patterns designed from the proposed procedure can effectively retain the information of the original full-color images. In particular, with the designed CFA patterns, high quality demosaicking can be achieved by using simple and efficient linear filtering operations in the polyphase domain. The visual qualities of the reconstructed images are competitive to those obtained by the state-of-the-art adaptive demosaicking algorithms based on the Bayer pattern. For future research, we plan to explore several variations on the proposed scheme, including adaptive demosaicking for improved visual quality, and luminance/chrominance weighting to exploit the fact that the human visual system is more sensitive to the luminance channel than to the chrominance channels.

\section{REFERENCES}

[1] Ramanath, R., Snyder, W. E., Bilbro, G. L., and Sander III, W. A., "Demosaicking methods for Bayer color arrays," J. Electron. Imag. 11, 306-315 (Jul. 2002).

[2] Gunturk, B. K., Glotzbach, J., Altunbasak, Y., Schafer, R. W., and Mersereau, R. M., "Demosaicking: Color filter array interpolation," IEEE Signal Process. Mag. 22, 44-54 (Jan. 2005). 

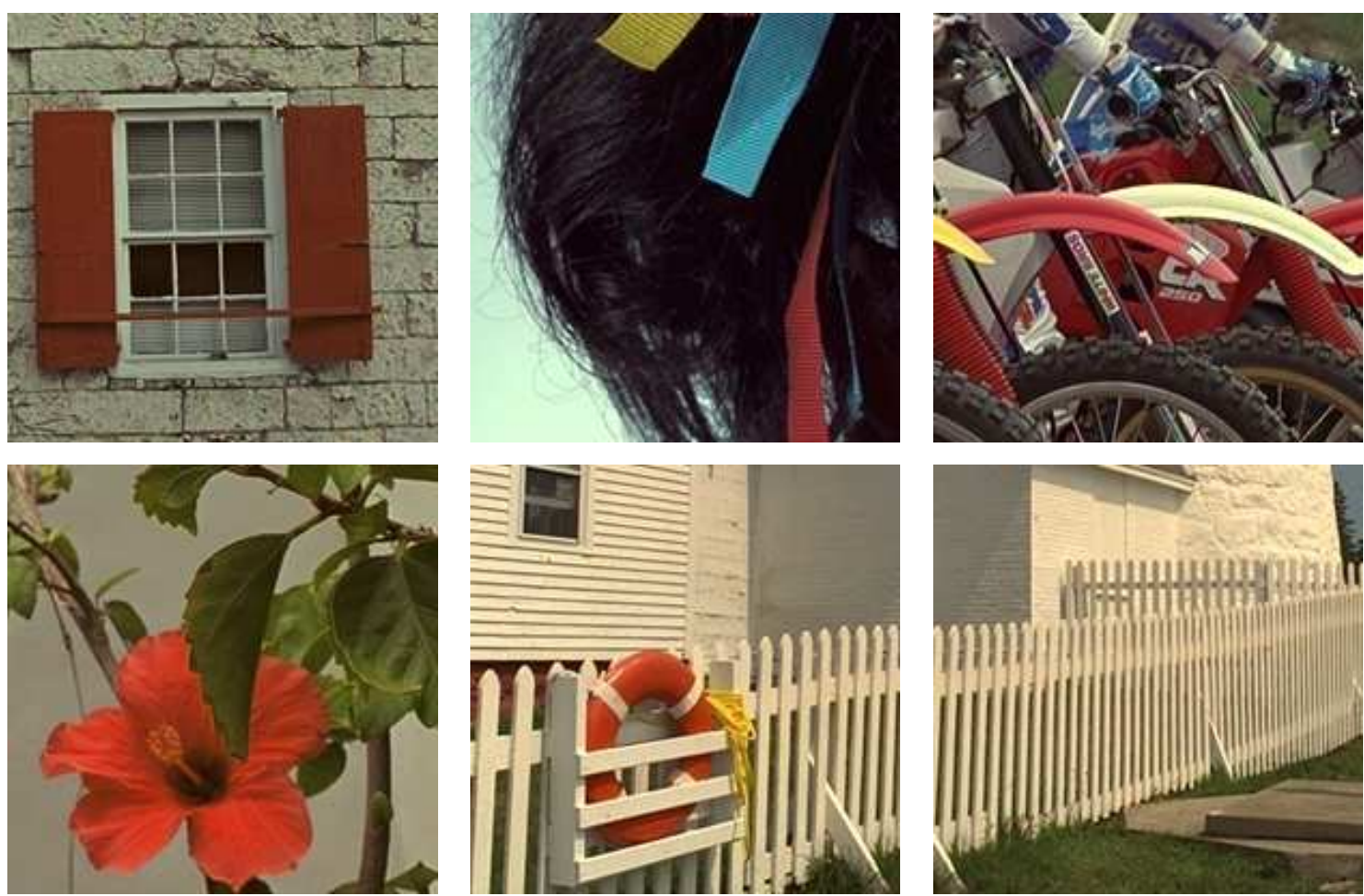

Figure 4. Detailed portions of six color images reconstructed from the 4-by-4 CFA patterns designed by the proposed scheme. The demosaicking algorithm uses simple nonadaptive 5-by-5 linear filtering in the polyphase domain.

[3] Li, X., Gunturk, B., and Zhang, L., "Image demosaicing: A systematic survey," in [Visual Communications and Image Processing 2008], Pearlman, W. A., Woods, J. W., and Lu, L., eds., Proc. SPIE 6822 (Jan. 2008).

[4] Bayer, B. E., "Color imaging array." U.S. Patent 3971065 (Jul. 1976).

[5] Lukac, R. and Plataniotis, K. N., "Color filter arrays: Design and performance analysis," IEEE Trans. Consum. Electron. 51, 1260-1267 (Nov. 2005).

[6] Kijima, T., Nakamura, H., Compton, J. T., and Hamilton, J. F., "Image sensor with improved light sensitivity." U.S. Patent 20070177236 (Aug. 2007).

[7] Hirakawa, K. and Wolfe, P. J., "Spatio-Spectral color filter array design for optimal image recovery," IEEE Trans. Image Process. 17, 1876-1890 (Oct. 2008).

[8] Taubman, D., "Generalized Wiener reconstruction of images from color sensor data using a scale invariant prior," in [Proc. IEEE Int. Conf. on Image Proc.], (Sep. 2000).

[9] Trussel, H. J. and Hartwig, R. E., "Mathematics for demosaicking," IEEE Trans. Image Process. 11, 485-492 (Apr. 2002).

[10] Chaix de Lavarène, B., Alleysson, D., and Héraut, J., "Practical implementation of LMMSE demosaicing using luminance and chrominance spaces," Computer Vision and Image Understanding 107(1-2), 3-13 (2007).

[11] Hotelling, H., "Analysis of a complex of statistical variables into principle components," J. Educ. Psychol. 24, 417-441, 498-520 (1933).

[12] Lu, Y. M. and Vetterli, M., "A quantitative theory for optimal color filer array design." in preparation (2008).

[13] Nocedal, J. and Wright, S., [Numerical Optimization], Springer, New York, NY (1999). 